\title{
Research Paper \\ Corona Anxiety in Nurses: The Predictive Role of Perceived Social Support and Sense of Coherence
}

\author{
*Sanaz Eyni ${ }^{1}\left(\mathbb{1}\right.$, Matineh Ebadi $^{1}$ (), Zohreh Hashemi² ${ }^{2}$
}

1. Department of Psychology, Faculty of Educational Sciences and Psychology, University of Mohaghegh Ardabili, Ardabil, Iran. 2. Department of Psychology, Faculty of Humanities, University of Maragheh, East Azerbaijan, Iran.

\begin{tabular}{|c|c|}
\hline $\begin{array}{l}\text { Use your device to scan } \\
\text { and read the article online }\end{array}$ & ditation Eyni S, Ebadi M, Hashemi Z. Corona anxiety in Nurses: The Predictive Role of Perceived Social Support and \\
\hline 口ifing & $\begin{array}{l}\text { Sense of Coherence. Iranian Journal of Psychiatry and Clinical Psychology. 2020; 26(3):320-331. http://dx.doi.org/10.32598/ } \\
\text { ijpcp.26.3436.1 }\end{array}$ \\
\hline 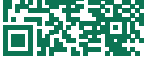 & doi'http://dx.doi.org/10.32598/ijpcp.26.3436.1 \\
\hline
\end{tabular}

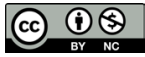

Received: 18 May 2020

Accepted: 13 Sep 2020

Available Online: 01 Dec 2020

Key words:

Coronavirus, Sense

of coherence, Social

support, Nurse

\begin{abstract}
A B S TRACT
Objectives Healthcare workers involved in the fight against corona are at high risk for depression and anxiety; therefore, this study aimed to predict Corona anxiety in nurses based on perceived social support and a sense of coherence.

Methods The research method was descriptive and correlational. The present study's statistical population consisted of all nurses working in hospitals and clinics in Rasht, employed in 2020, and 200 people participated in the survey online and through virtual networks. Data collection tools included the Corona anxiety Scale, Multidimensional Scale of Perceived Social Support, and Sense of Coherence Inventory(SOC)13 Scale. Data were analyzed using the Pearson correlation test and simultaneous regression analysis using SPSS V. 23 software.

Results Corona anxiety in nurses had a negative and significant relationship with perceived social support $(\beta=-0.581 ; P<0.03)$ and sense of coherence $(\beta=-0.672 ; P<0.001)$. Perceived social support and a sense of coherence of $42 \%$ of variance predicted Corona anxiety scores in nurses $(P<0.01)$.

Conclusion Therefore, perceived social support and a sense of coherence play an essential role in nurses' Corona anxiety. Therefore, educating nurses to increase the understanding of cohesion to empower and implement understandable support programs is recommended.
\end{abstract}

\section{Extended Abstract}

\section{Introduction}

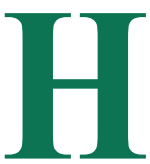

ealthcare workers involved in the fight against corona are at high risk for depression and anxiety. Nurses are one of the most critical elements of health care because the main burden of providing services and responsibility for human life and health is on them, so they are continually experiencing severe psychological stress [1]. These days, the most common emotional reaction that nurses are dealing with is anxiety because Covidase 19 has caused concern among people, especially in the affected countries. Covid 19 is a severe threat to public health $[5,4]$.

One of the essential sources of adaptation in stressful situations is the amount of support and attention received from loved ones. Another variable is the sense of coherence. The sense of cohesion refers to an individual's ability to understand a particular situation and to make effective use of available resources that allows the individual to use adaptive coping strategies [23]. Therefore, due to the increased -

* Corresponding Author:

Sanaz Eyni, PhD.

Address: Department of Psychology, Faculty of Educational Sciences and Psychology, University of Mohaghegh Ardabili, Ardabil, Iran.

Tel: $+98(914) 455128$

E-mail: sanaz.einy@yahoo.com 
prevalence of the virus and the increase in the number of nurses' deaths, which can be a stressor for other nurses, it is necessary to pay attention to nurses' health.

As one of the top 10 countries with the highest pollution levels in Iran, evaluating nurses' knowledge of Quaid 19 could be a practical step in controlling the disease [9]. Therefore, this study aimed to predict the Corona anxiety in nurses based on perceived social support and coherence.

\section{Method}

This study was descriptive-correlational in terms of practical purpose and how to collect data. The study's statistical population consisted of all nurses working in hospitals and clinics in Rasht employed in 2020. The sample consisted of 200 nurses who participated in the survey online and through virtual networks. This method was chosen due to limited traffic when the risk of corona infection became serious in Iran. The following tools were used to collect data: Corona anxiety Scale (Alipour et al. 2020) [20], Multidimensional Scale of Perceived Social Support (Zimet et al., 1988) [38], and Sense of Coherence Inventory-(SOC)13 Scale (Antonovsky, 1987) [40].

The research method was as follows: the questionnaires were designed online and distributed in cyberspace, including (Telegram, WhatsApp, and Instagram) among nurses, of which 200 responses were refined and selected. Data analyzed using the Pearson correlation test and structural equation modeling with SPSS V. 23 software. Corona anxiety was considered as a criterion variable, perceived social support, and a sense of coherence as predictor variables.

\section{Results}

The statistical sample was 200 nurses (Mean \pm SD age of $35.5 \pm 2.5$ years), ranging from $25-45$. Of them, 113 (56.5\%) were women, and 87 (43.5\%) were men. Coronavirus anxiety in nurses was perceived to have a negative and significant relationship with perceived social support and a sense of coherence $(\mathrm{P}<0.01)$.
Regression analysis was used to explain the perceived share of perceived social support and the sense of coherence in predicting Corona anxiety in nurses. The results are as follows: before the regression analysis, a preliminary study was performed to evaluate regression assumptions. The values obtained for Tolerance and VIF indicated that no incorrect belief they had, have not been violated. Because the Tolerance value of all variables is more than 0.1 and the VIF value of the variables is less than 10. The Watson Camera Test was also used to investigate the independence of errors, indicating that the errors were independent.

The multiple regression analysis results in predicting Corona anxiety in the perceived social support and sense of coherence. The predictor variables of $42 \%$ of variance indicate Corona anxiety scores among nurses (F:263/163) are signific antly $<0.01$. The regression coefficients also show that $\mathrm{p}$ e rceived social support $(\mathrm{P}=0.036=7.46)$ and $(\mathrm{P}=0.001=\mathrm{P}=0.73)$ inversely predict Corona anxiety in nurses. They do. Beta values showed that cohesiveness contributed most to predicting Corona anxiety in nurses.

\section{Discussion and Conclusion}

The correlation coefficients showed that nurses' Corona anxiety had a negative and significant relationship with perceived social support. The regression analysis results also showed that perceived social support of variance explains Corona anxiety scores in nurses. The findings, therefore, support the link between perceived social support and Corona anxiety in nurses. To illustrate the result, perceived social support is essential in predicting individuals' physical and psychological health from childhood to adulthood and as a shock shield. The sticker acts against stress [16].

Regarding the relationship between Corona artery cohesion and nurses' anxiety, the correlation coefficients showed that cohesiveness score was negatively related to nurses' Corona anxiety. Therefore, nurses who have a strong sense of cohesion expect their stimuli to be predictable in the future or show clear and relevant behavior when confronted with a stressful event. It reduces the severity of the clinical

Table1. Research solidarity solidarity matrix

\begin{tabular}{cccc}
\hline Variable & $\mathbf{1}$ & $\mathbf{2}$ & $\mathbf{3}$ \\
\hline 1. Percieved Social Support & 1 & & \\
2. Sense of Coherence & $0.438^{* *}$ & 1 & 1 \\
\hline 3. Corona Virus Anxiety & $-0.516 .0^{* *}$ & $-0.521^{* *}$ & \\
\hline
\end{tabular}

$* * \mathrm{P}<0.01$.

Iranian Journal of
PSYCHIATRY AND CLINICAL PSYCHOLOGY 
features of Corona anxiety. In general, the perceived social support and a sense of cohesion are associated with Corona anxiety in nurses and can predict how a person will react to anxiety. Therefore, training nurses to empower them in these areas is recommended.

\section{Ethical Considerations}

Compliance with ethical guidelines

All ethical principles are considered in this article. The participants were informed about the purpose of the research and its implementation stages. They were also assured about the confidentiality of their information and were free to leave the study whenever they wished, and if desired, the research results would be available to them.

Funding

This research did not receive any specific grant from funding agencies in the public, commercial, or not-forprofit sectors.

Authors' contributions

Writing - original draft, and writing - review \& editing: Sanaz Eyni; Investigation, data collection: Matineh Ebadi; Data analysis: Zohreh Hashemi.

\section{Conflicts of interest}

The authors declared no conflict of interest. 


\title{
اضطراب كرونا در يرستاران: نقش ييشبين حمايت اجتماعى ادراكشده وحس انسجام
}

\author{
(1) 'ساناز عينى' (1)، متينه عبادى' (1) زهره هاشمى \\ ا. كروه روانشناسى، دانشكده علوم تربيتى و روانشناسى، دانشكاه محقق اردبيلى، اردبيل، ايران.

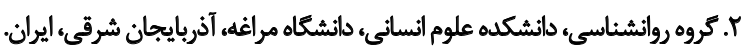

\begin{abstract}
حكيد

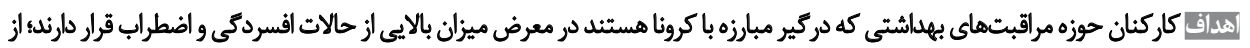

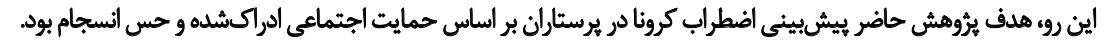

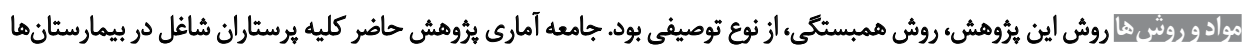

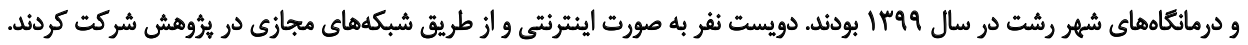

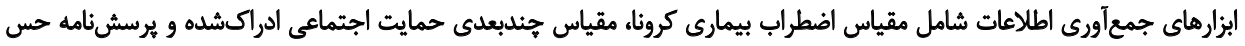

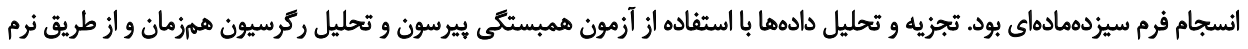

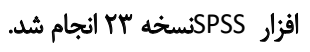

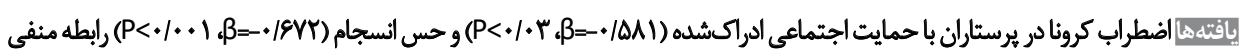

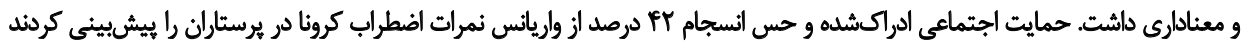
$(\mathrm{P}<\bullet 1 \cdot 1)$

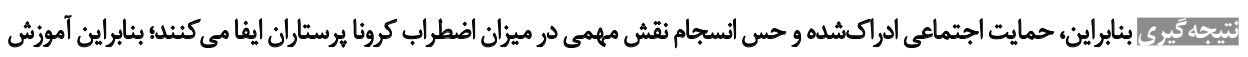

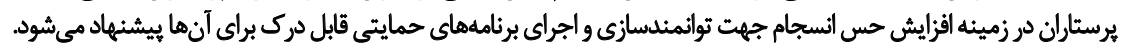

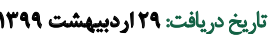

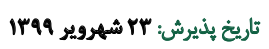

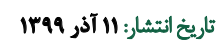

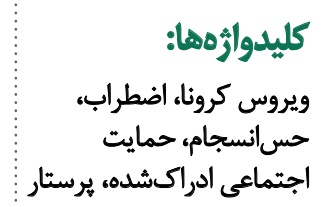

كليدوازوها:

ويروس كروناء اضطراب،

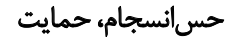

اجتماعى ادراكشده، برستار
كستردكى شيوع كوويد 19 در جهان و ميزان تلفات اين بيمارى

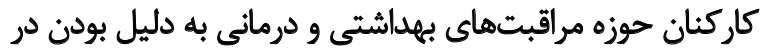

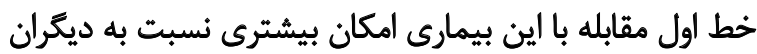

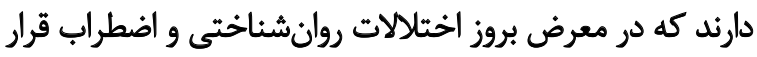

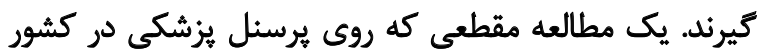

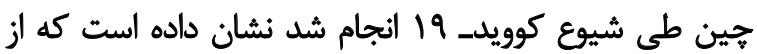

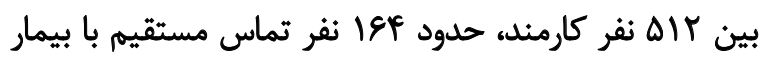

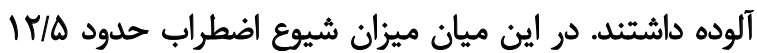

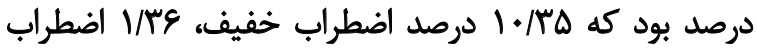

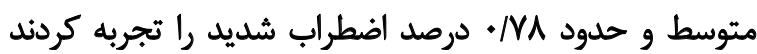

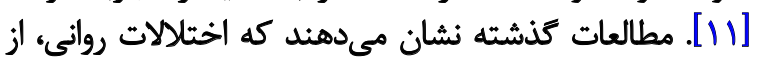

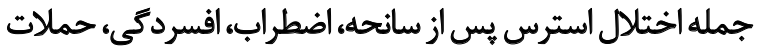

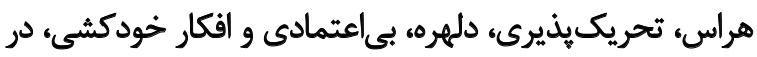

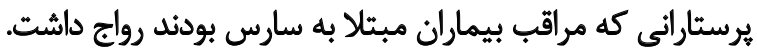

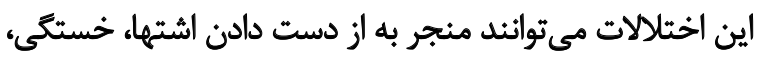

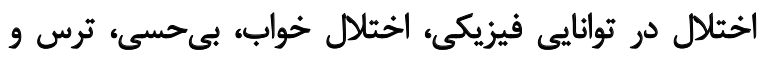

dalês

يكى از مهمهرين عناصر مراقبت سلامت، يرستاران هستند؛ زيرا

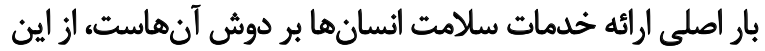

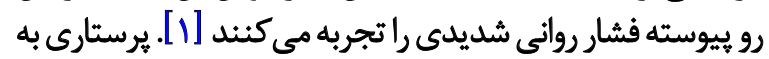

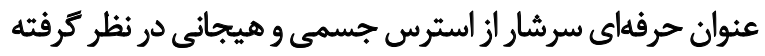

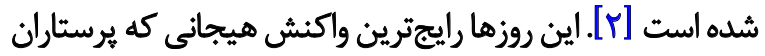

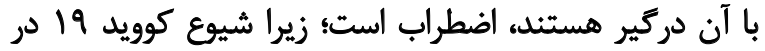

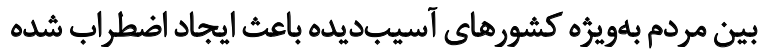

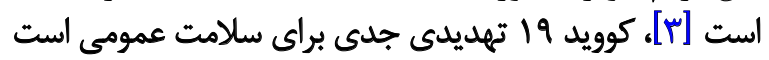

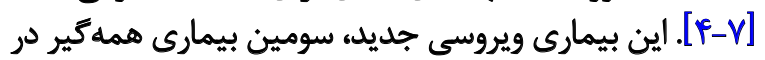

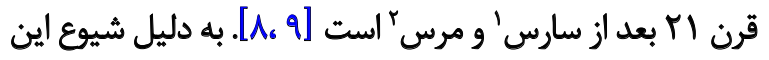

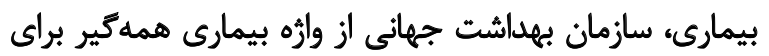

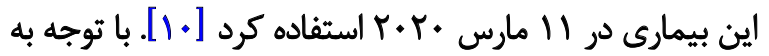

1. SARS

2. MERS 
افراد داراى حس انسجام قوى در مواجهه با عوامل استرسزاه

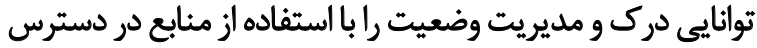

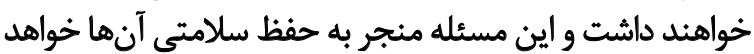

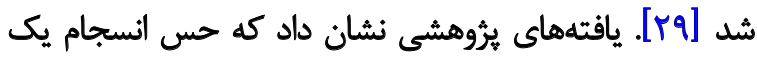

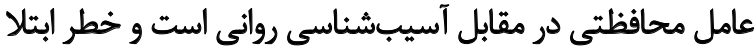

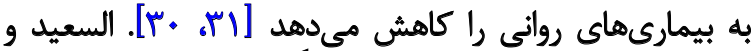

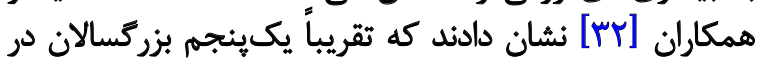

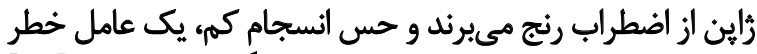

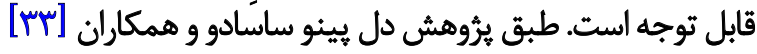

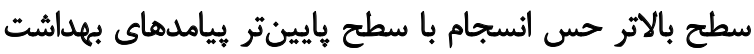

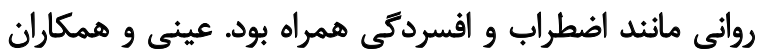

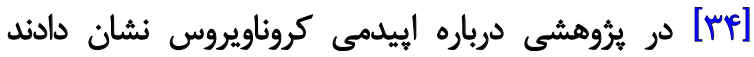

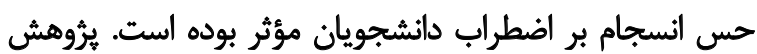

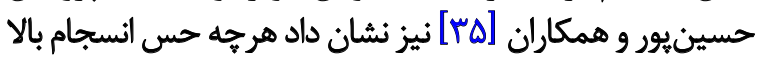
باشد، ميزان اضطراب هايينتر خواهد بود.

با توجه به شيوع بيشتر ويروس و همجنين افزايش تعداد

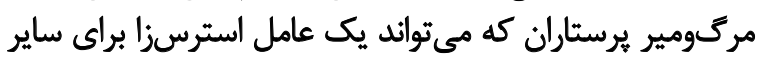

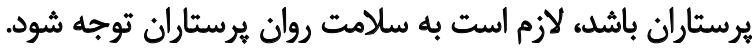

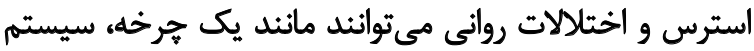

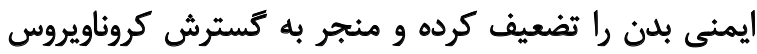

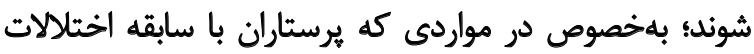

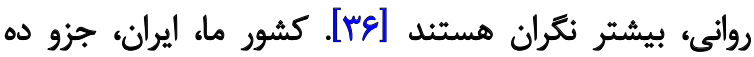

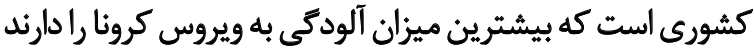

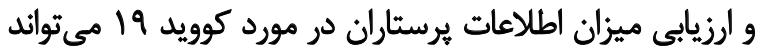

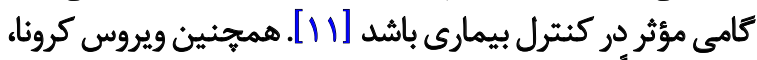

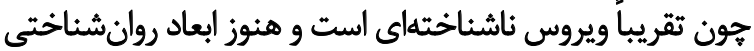

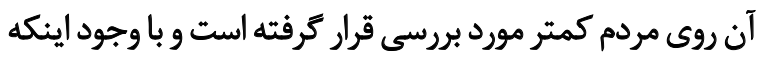

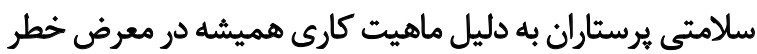

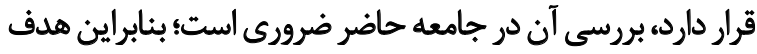

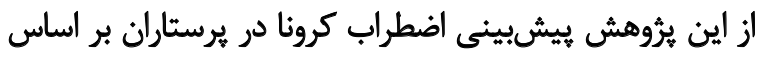

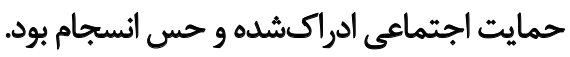
و

اين مطالعه از نظر هدف كاربردى و روش كردآورى دادهها

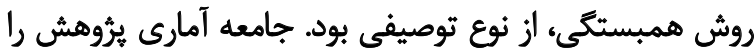

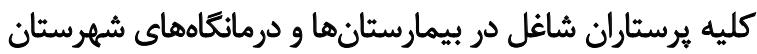

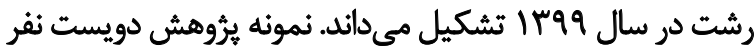

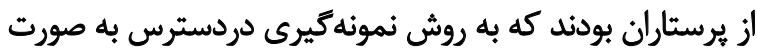

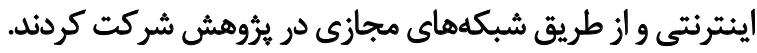

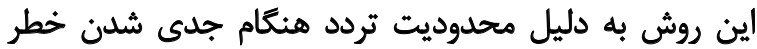

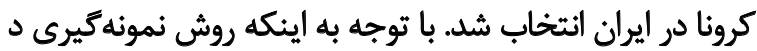

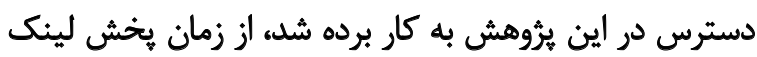

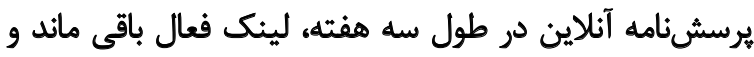

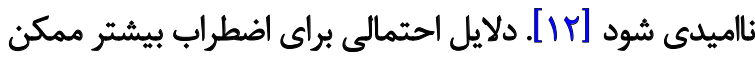

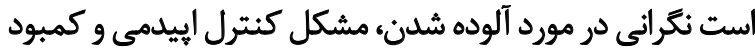

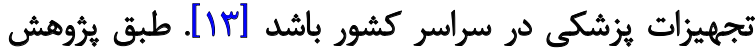

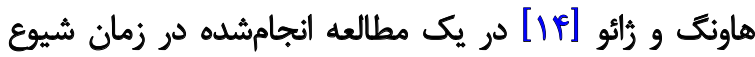

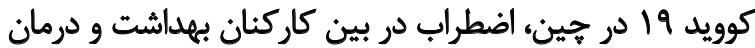

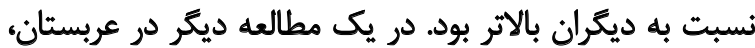

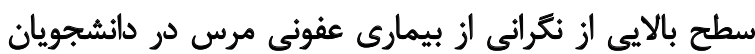

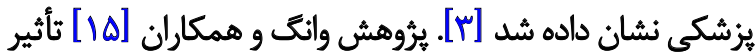

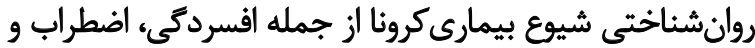

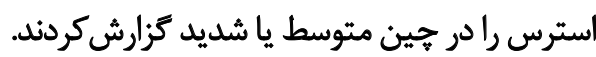

از جمله منابع مههم سازكارى در شرايط تنش سزا ميزان حمايت

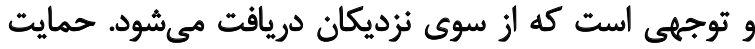

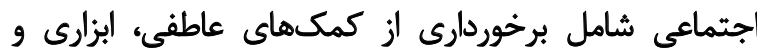

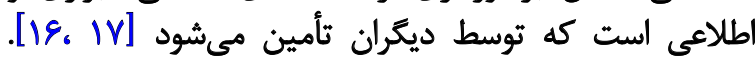

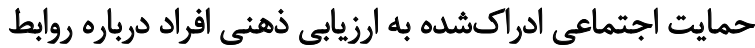

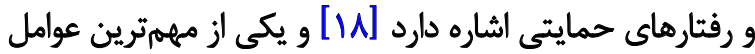

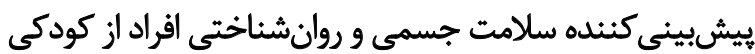

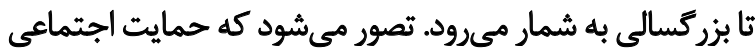

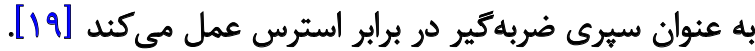

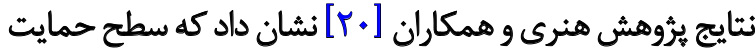

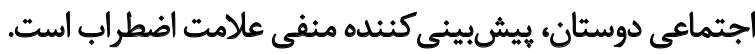

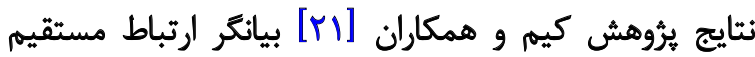

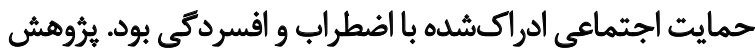

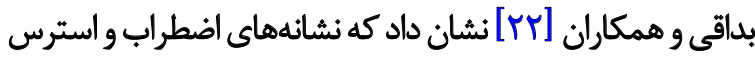

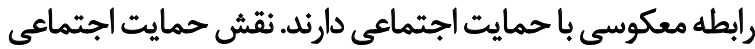

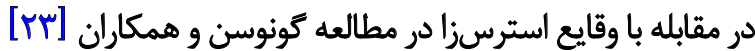

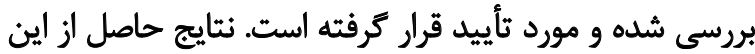

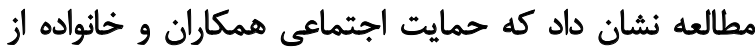

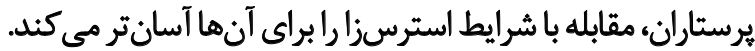

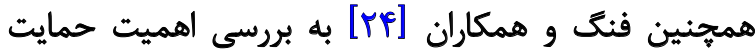

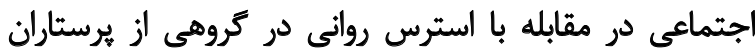

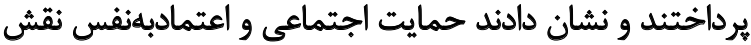
مهمى در مقابله با استرسهان داند روايت إنتى داشته است.

زتغير ديكرى كه منعكس كنئده جهت كيرى كلى فرد به به إنه

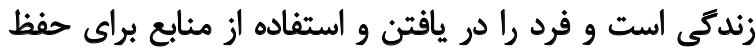

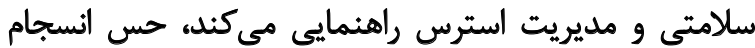

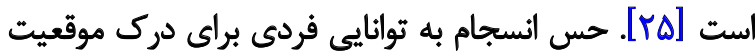

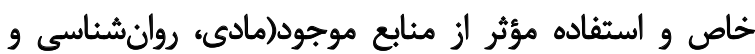

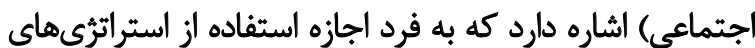

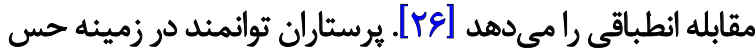

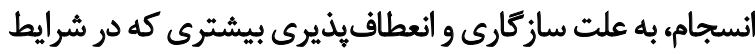

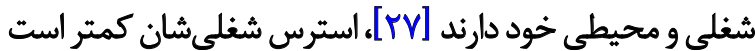

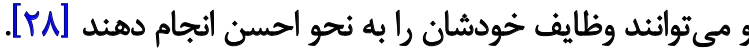


جدول ا. توصيف متغيرهاى يُروهش

\begin{tabular}{|c|c|c|c|c|}
\hline كشيدكّى & جولَّى & واريانس & ميانكين +انحراف معيار & هثغيرها \\
\hline.$/ M r$ & $M \cdot r$ & I.NTAV & $W M+ \pm 1 \cdot / P \cdot \Delta$ & اضطراب كروناويروس \\
\hline $.1 \cdot m$ &.$/ 1 e q a$ & MI/UV & $11 / M^{2} \pm$ /8/9 & عالاتم رواثى \\
\hline - mar & I/YAI & PTPEI & $q / 8 \mid \pm 8 / \Delta)^{\prime q q}$ & علائم جسمانى \\
\hline.$- / 1 \mathrm{~W}$ & $-.18 \mathrm{AV}$ & larplere & E./AVEIY/TNE & حمايت اجتماعى ادراكشده \\
\hline.$- / M T r$ &.$- / A r q$ & rVliar & $M V / A \pm \Delta / M I I$ & حمايت خانواده \\
\hline.$- /$ ret &.$- / 11 f$ & rIMIV & Wreteles. & حمايت دوستان \\
\hline.$- / T \Delta P$ & $-\left.\cdot(A)\right|^{2}$ & $r N M+r$ & $r M / M T \pm \Delta / M T$. & حمايت ساير أفراد \\
\hline.$- / 8 N$ & - - AAFQ & PNVTY & $V \cdot / 8 \mid \pm \& / 4$. & حس انسجام \\
\hline$+/ f+9$ &.$- / A M F$ & $9 / M+r$ & $r V / r q \pm w / \cdot W$ & ادراكئذيرى \\
\hline-.1 .18 &.$- / 014$ & $\Delta / \Lambda \cdot \varepsilon$ & $r M / r \pm T / Y+q$ & كنترليذيرى \\
\hline .1180 &.$- /$ tra & $\Delta / r+\Lambda$ & YV/DQ \pm Y/TAY & معنى دارى \\
\hline
\end{tabular}

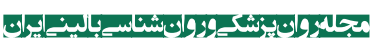

خانواده، دوستان و افراد مهم در زند ماكى در ليكرت هفت كزينهاى

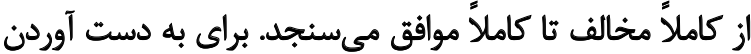

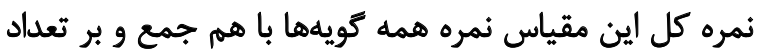

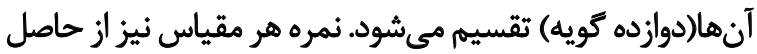

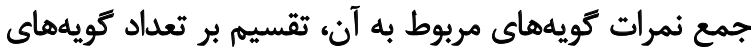

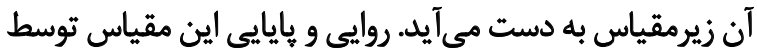

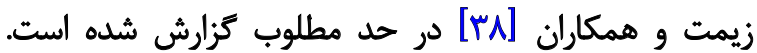

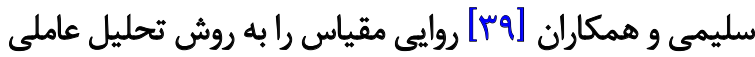

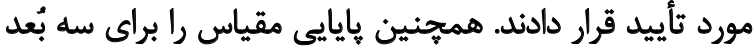

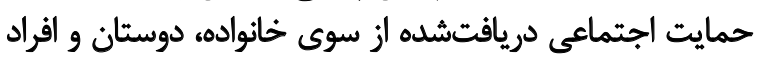

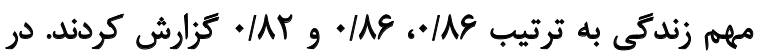

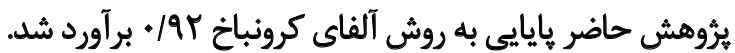
يرسشنامه حس انسجام فرم سيزدهمادهاي:ه فرم كوتاه يرسشنامه حس انسجام توسط آنتونووسكى در سال

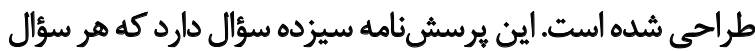

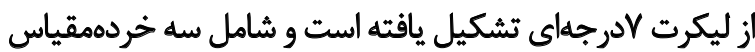

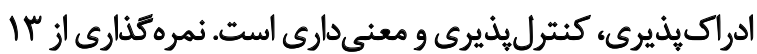

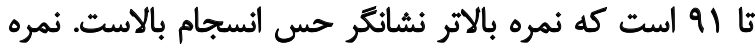

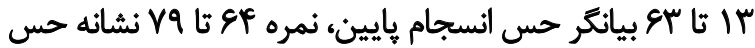

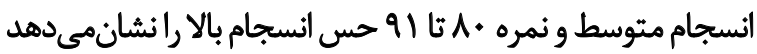

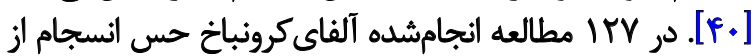

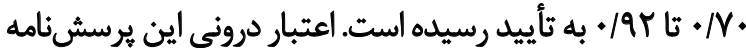

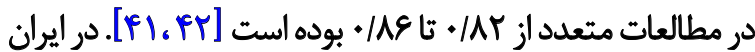

5. Sense of Coherence Inventory-(SOC) 13 scale
در طول اين مدت، دويست يرسشنامه با حذف موارد ناقص در اختيار يثوهشگران قرار كرفت.

$$
\text { أبزأرهاى تردآورى دادهها }
$$

يرسشنامه اضطراب كرونا ويروس": اين ابزار جهت سنجش إنش

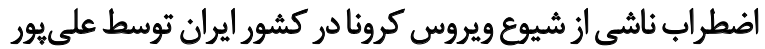

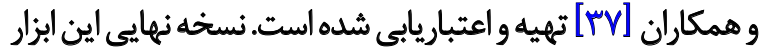

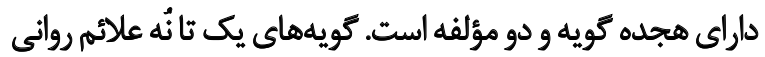

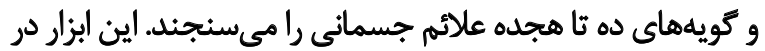

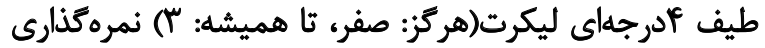

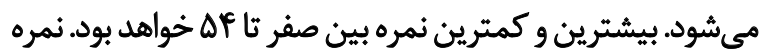

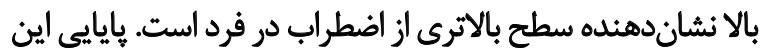

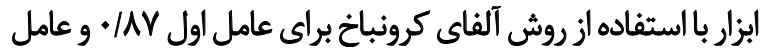

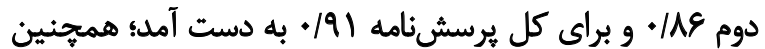

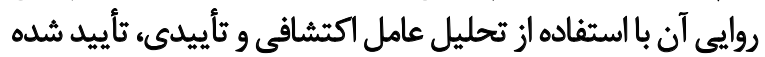

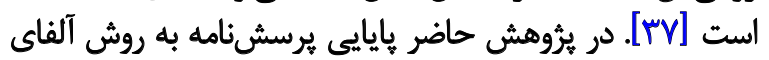

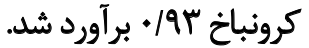

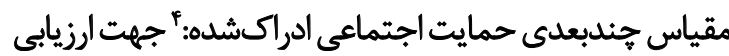

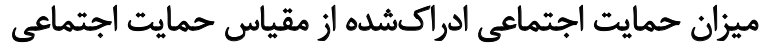

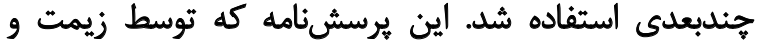

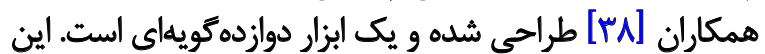

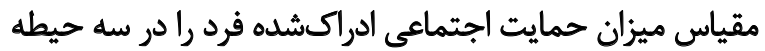

4. Multidimensional Scale of Perceived Social Support (MSPSS) 
جدول Y. ماتريس همبستكى متغيرهاى بئرهش

\begin{tabular}{|c|c|c|c|}
\hline$r$ & r & 1 & مثغير \\
\hline & & 1 & ا. حمايت اجتماعى ادراكشده \\
\hline & 1 &.$/ R T \Lambda^{* *}$ & r. حس انسجام \\
\hline 1 & $-\cdot(\Delta Y)^{* *}$ & $-+\left.|0|\right|^{* * *}$ & r. اضطراب كرونا ويروس \\
\hline
\end{tabular}

جدول r. نتايج تحليل ركرسيون اضطراب كرونا ويروس در يرستاران بر اساس حمايت اجتماعى ادراكشده و حس انسجام

\begin{tabular}{|c|c|c|c|c|c|c|c|c|}
\hline$t(p)$ & Beta & B & SE & Sig. & $\mathbf{F}$ & $\mathbf{R}^{2}$ & متغيرهاي ييشيين & متغير ماكى \\
\hline & & & & $\%$ & $|8 \% / r g|$ & . & & \\
\hline 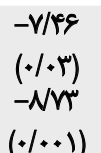 & $\begin{array}{l}-.|\Delta A| \\
-. \mid 8 x\end{array}$ & $\begin{array}{l}-. / \Delta \mathrm{M}^{2} \\
-. / 9 \mathrm{gr}\end{array}$ & $\begin{array}{l}. / \cdot A \mu \\
. V H\end{array}$ & & & & حمايت اجتماعي ادراكشده & اضطراب كرونا در برسثاران \\
\hline
\end{tabular}

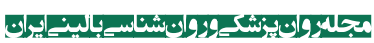

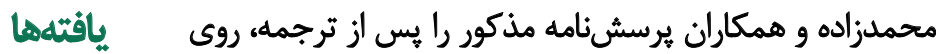

نمونه آمارى مورد مطالعه دويست برستار با متوسط سن دال

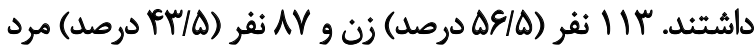

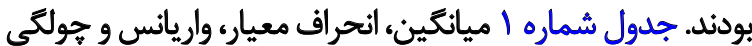

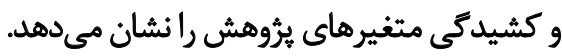

مطابق جدول شماره ا، مقدار جولكى مشاهدهشده براى

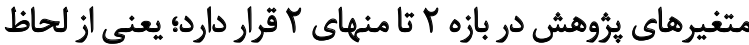

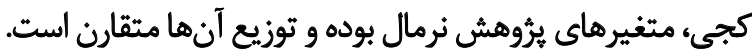

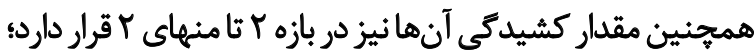
اين نشان ميدهد توزيع متغيرهاى مورد مطالعه از كشيديدكي

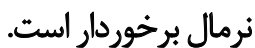

به منظور بررسى رابطه بين متغيرهاى يرؤهش از ضريب

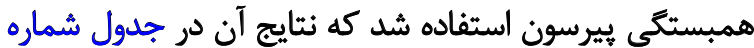

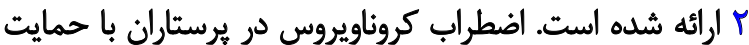

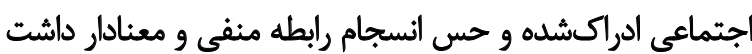
( $(P<+1 \cdot 1)$

جهت تبيين سهرم حمايت اجتماعى ادراكشده و حس انسجام

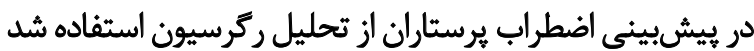

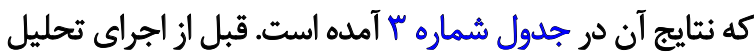

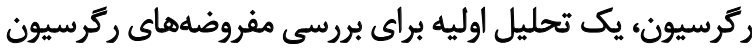

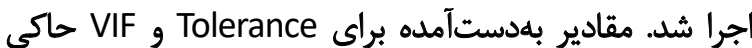

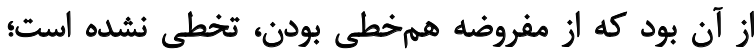

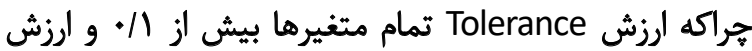

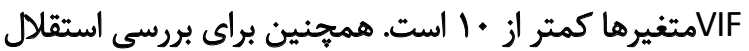

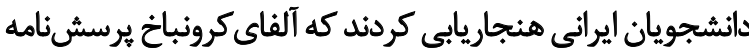

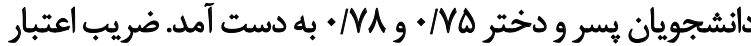

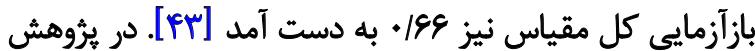

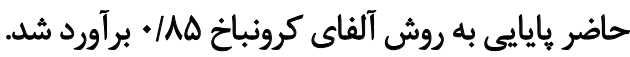

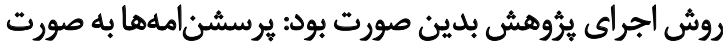

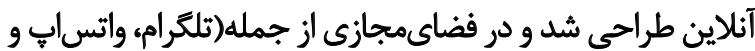

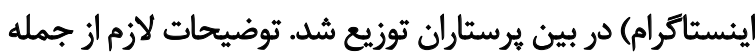

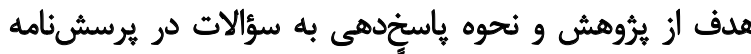

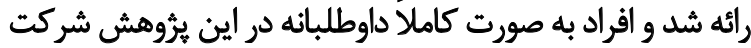

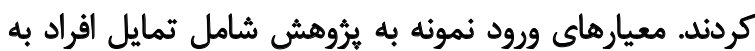

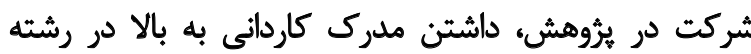

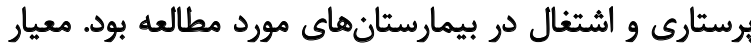

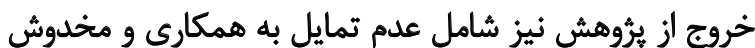

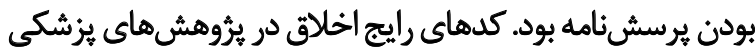

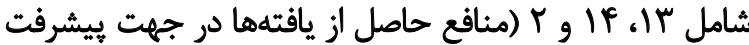

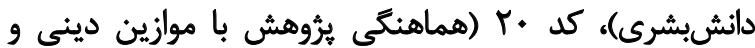

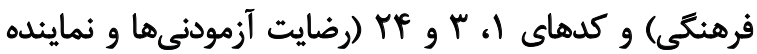

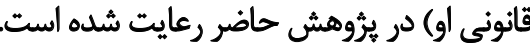

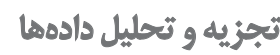

تجزيه و تحليل دادهها با استفاده از آزمون همبستخى يُيرسون

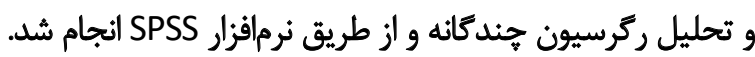

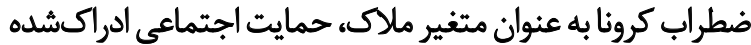
و حس انسجام به عنوان متغيرهاى بيشيش منين الحاظ شدند. 
افراد داراى سطوح بالاى حمايت اجتماعى ادراكشده مطمئن

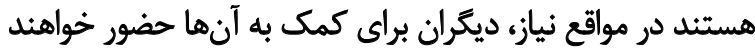

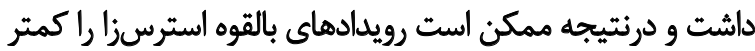

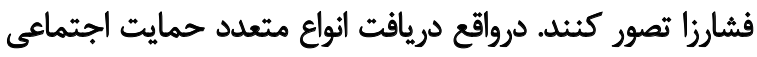

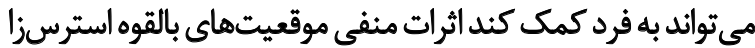
را به طور مستقيم حذف كند يا دائ دست كم آنها

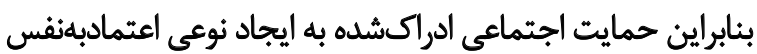

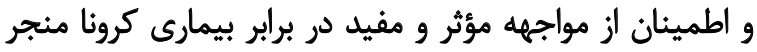

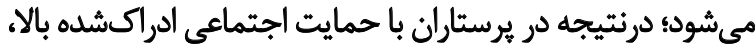

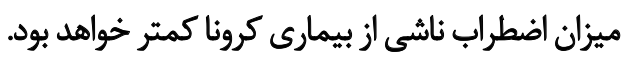
درارتباط بار ابطه بين حس انسجامو اضطراب كرونادر يرستاران،

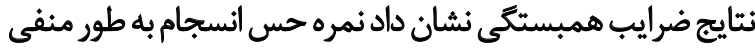

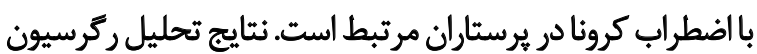

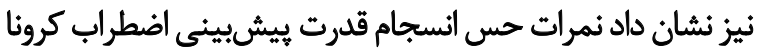

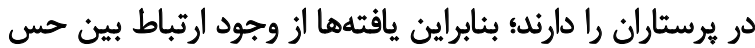

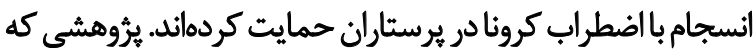

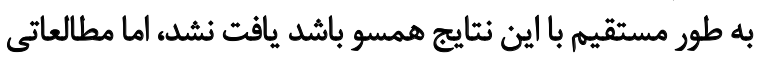

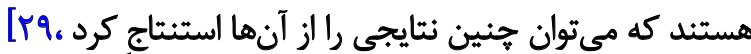

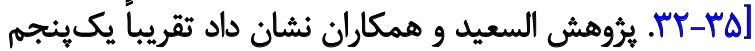

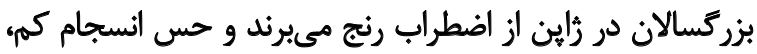

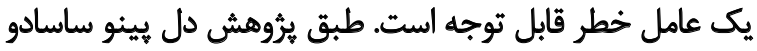

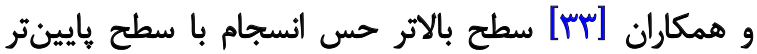

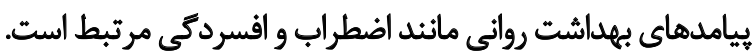

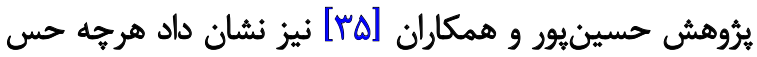

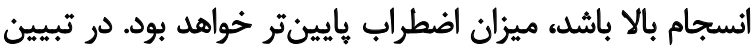

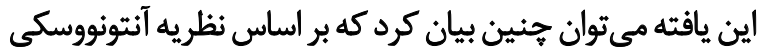

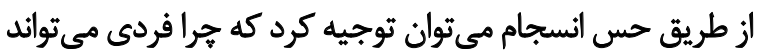

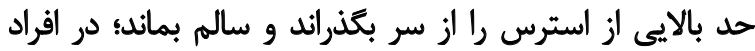

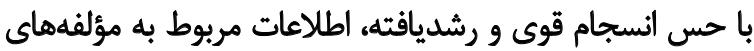

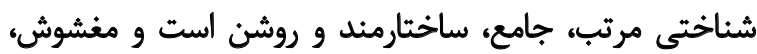

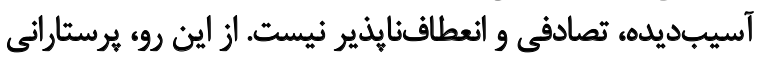

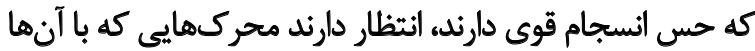

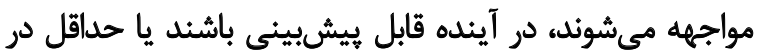

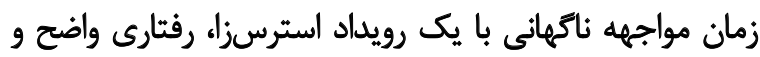

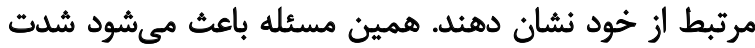

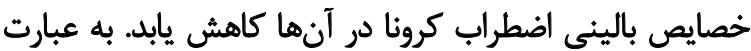

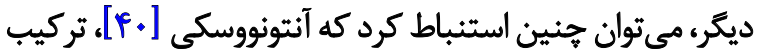

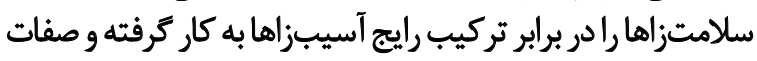

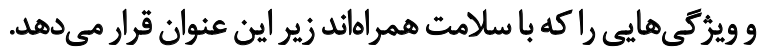

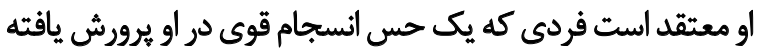

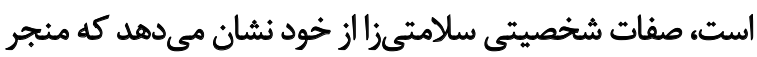

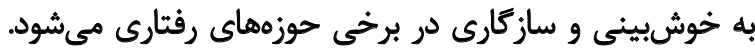

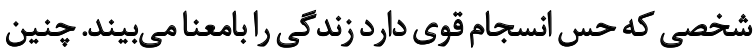

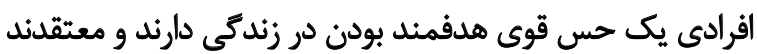

خطاها از آزمون دوربين واتسون استفاده شد كه ميزان آن نشان

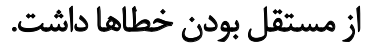

بر اساس نتايج جدول شماره ب، نتايج تحليل ركرسيون

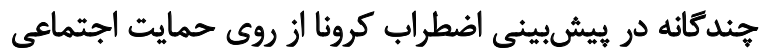

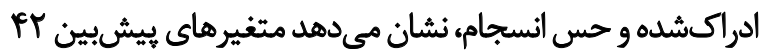

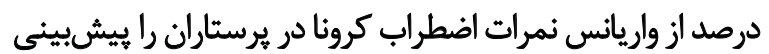

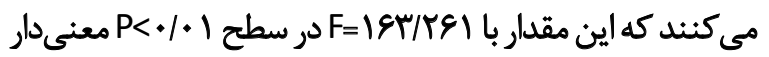

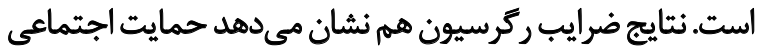
ادراكشده (P<

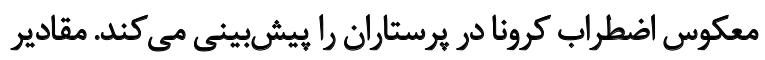

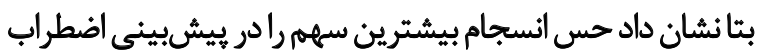
كرونا در يرستاران دارد.

ثب

يُروهش حاضر با هدف بررسى نقش حمايت اجتماعى

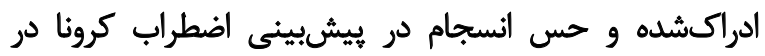

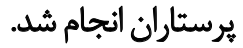

نتايج ضرايب همبستكى نشان داد اضطراب كرونا در برستاران

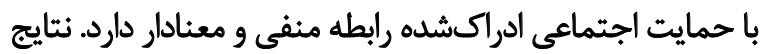

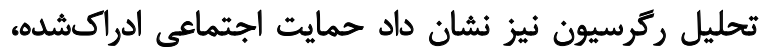

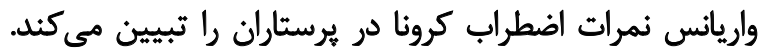

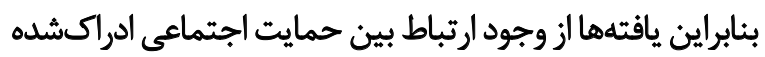

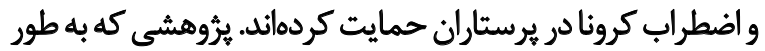

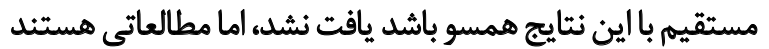

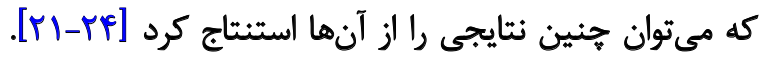

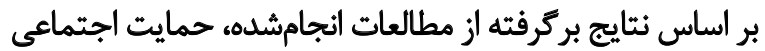

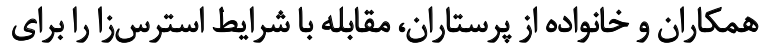

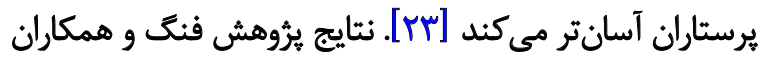

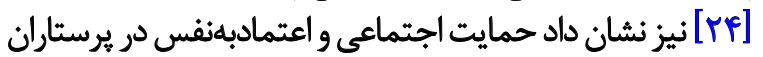

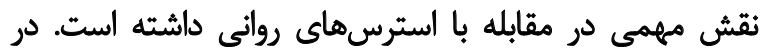

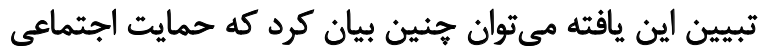

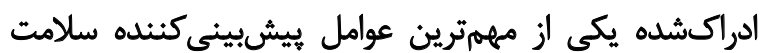

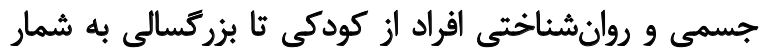
مى آيد و به عنوان سيرى در برابر استرس عمل مي كندا فئد اين يافته تقويت كننده نظريه مستقيم حمايت اجتماعي است؛

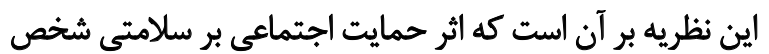

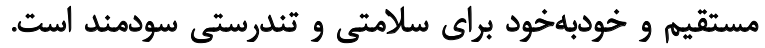

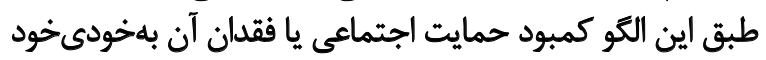

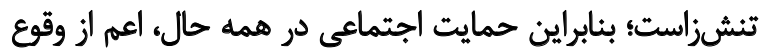

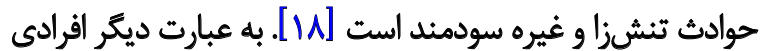

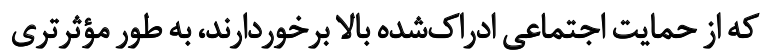

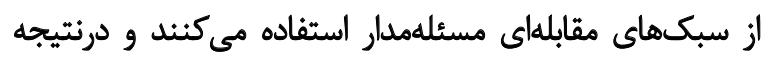
آشفتكى كمتر و بهزيستى و سلامت بالاترى را كزارش مى كنيند. 
شد، با مراجعه به كلينيكهاى رواندرماني، با دريافت برنامه هائى

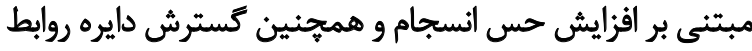

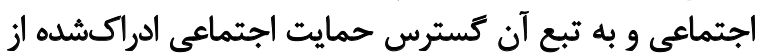
سوى ديكران با اين اضطراب مقابله كنثد.

مالاحظاث اخلاقى

\section{ييروى أز الصول اخلاق يؤوهش}

كليهى اصول اخلاقى در اين مقاله رعايت شده است. شركت

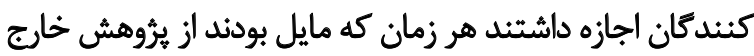

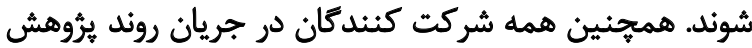

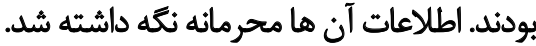

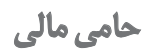

اين تحقيق هيج كونه كمك مالى خاصى از سوى سازمانهاى

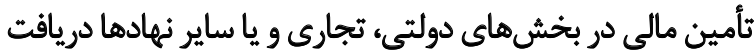

$$
\text { مشاركت نويسند مَان }
$$

نوشتن بيشنويس اصلى مقاله، بررسى و ويرايش: ساناز عينى؛

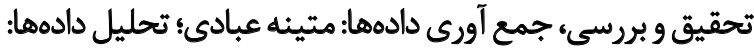

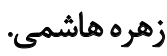

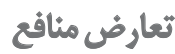

بنابر اظهار نويسندكانء اين مقاله تعارض منافع ندارد.
تجارب استرسزاى زندكى قابل فهم و درك هستند. افراد با حس

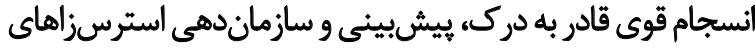

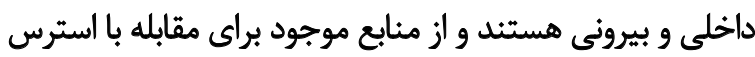

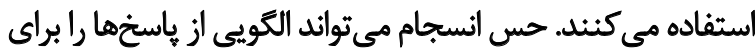

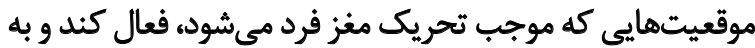

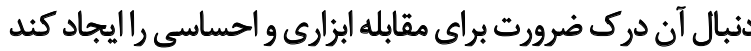

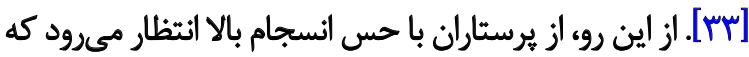

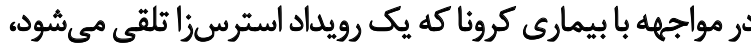

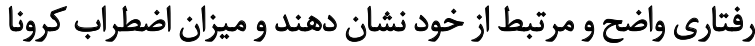

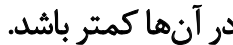

$$
\text { نتيجهنيرى }
$$

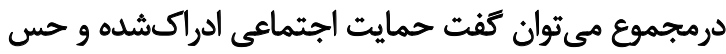

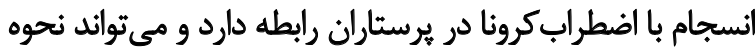

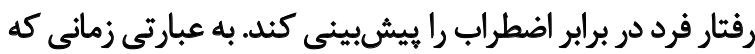

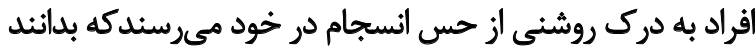

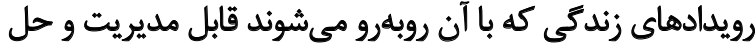

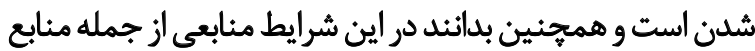

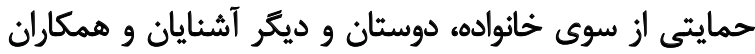

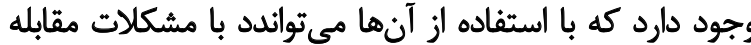

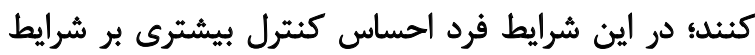

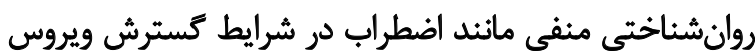

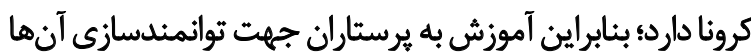
در اين زمينهها ييشنهاد مي أشيود.

يُروهش حاضر با محدوديتهايى نيز روبه رو بود؛ از جمله اينكه

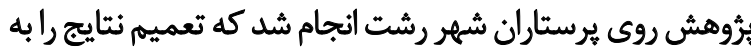

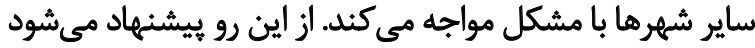

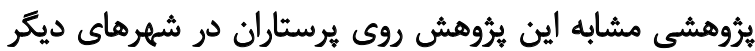

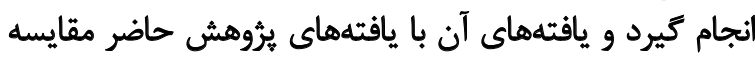

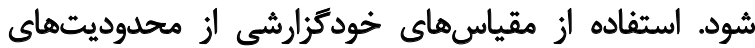

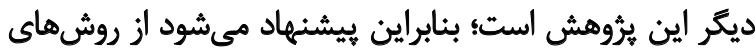

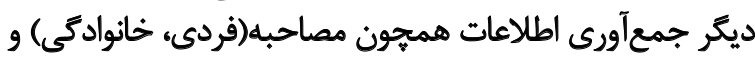

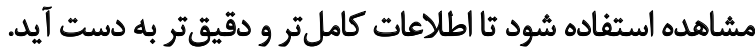

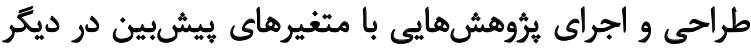

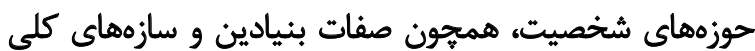

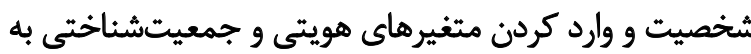

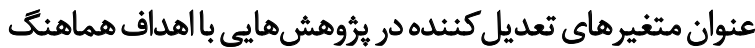

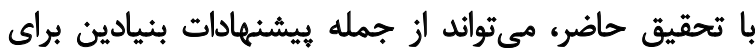

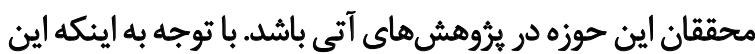

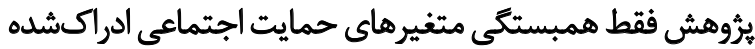

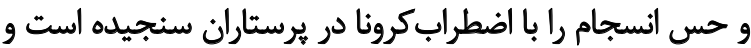

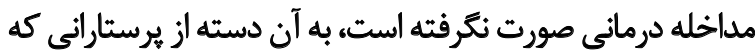

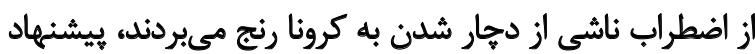




\section{Reference}

[1] Boustanipour L, Saberi H, Soheili A. [Prediction of level of aggression and interpersonal problems in Ed nurses based on their occupational stress (Persian)]. Nursing and Midwifery Journal. 2017; 15(6):478-87. http://unmf.umsu.ac.ir/browse.php?a id $=3257 \&$ sid $=1 \&$ slc_lang $=$ fa

[2] Brennan EJ. Towards resilience and wellbeing in nurses. British journal of nursing (Mark Allen Publishing). 2017; 26(1):43-7. [DOI:10.12968/bjon.2017.26.1.43] [PMID]

[3] Al-Rabiaah A, Temsah M-H, Al-Eyadhy AA, Hasan GM, AlZamil F, Al-Subaie S, et al. Middle East Respiratory SyndromeCorona Virus (MERS-CoV) associated stress among medical students at a university teaching hospital in Saudi Arabia. Journal of Infection and Public Health. 2020; 13(5):687-91. [DOI:10.1016/j. jiph.2020.01.005] [PMID] [PMCID]

[4] Jernigan DB. Update: Public health response to the Coronavirus Disease 2019 Outbreak-United States, February 24, 2020. MMWR. Morbidity and Mortality Weekly Report. 2020; 69(8):216-9. [DOI:10.15585/mmwr.mm6908e1] [PMID] [PMCID]

[5] Liao X, Wang B, Kang Y. Novel coronavirus infection during the 2019-2020 epidemic: Preparing intensive care units- the experience in Sichuan Province, China. European Journal Of Intensive Care Medicine. 2020; 46(2):357-60. [DOI:10.1007/s00134-02005954-2] [PMID] [PMCID]

[6] Arefi MF, Poursadeqiyan M. A review of studies on the COVID-19 epidemic crisis disease with a preventive approach. Work. 2020; 66(4):717-29. [DOI:10.3233/WOR-203218] [PMID]

[7] Poursadeqiyan M, Bazrafshan E, Arefi MF. Review of environmental challenges and pandemic crisis of Covid-19. Journal of Education and Health Promotion. 2020; 9:250 [DOI:10.4103/jehp. jehp_420_20] [PMID] [PMCID]

[8] Chang D, Lin M, Wei L, Xie L, Zhu G, Cruz CSD, et al. Epidemiologic and clinical characteristics of novel coronavirus infections involving 13 patients outside Wuhan, China. JAMA. 2020; 323(11):1092-3. [DOI:10.1001/jama.2020.1623] [PMID] [PMCID]

[9] Paules CI, Marston HD, Fauci AS. Coronavirus infectionsmore than just the common cold. JAMA. 2020; 323(8):707-8. [DOI:10.1001/jama.2020.0757] [PMID]

[10] Takian A, Raoofi A, Kazempour-Ardebili S. COVID-19 battle during the toughest sanctions against Iran. Lancet (London, England). 2020; 395(10229):1035-6. [DOI:10.1016/S01406736(20)30668-1]

[11] Brooks SK, Webster RK, Smith LE, Woodland L, Wessely S, Greenberg N, et al. The psychological impact of quarantine and how to reduce it: Rapid review of the evidence. Lancet. 2020; 395:912-20. [DOI:10.1016/S0140-6736(20)30460-8]

[12] Lai J, Ma S, Wang Y, Cai Z, Hu J, Wei N, et al. Factors associated with mental health outcomes among health care workers exposed to coronavirus disease 2019. JAMA Network Open. 2020; 3(3):e203976. [DOI:10.1001/jamanetworkopen.2020.3976] [PMID] [PMCID]

[13] Nemati M, Ebrahimi B, Nemati F. Assessment of iranian nurses' knowledge and anxiety toward Covid-19 during the current outbreak in Iran. Archives of Clinical Infectious Diseases. 2020; 15(COVID-19):e102848. [DOI:10.5812/archcid.102848]
[14] Huang Y, Zhao N. Generalized anxiety disorder, depressive symptoms and sleep quality during COVID-19 epidemic in China: A web-based cross-sectional survey. Psychiatry research. 2020; 288:112954. [DOI:10.1016/j.psychres.2020.112954] [PMID] [PMCID]

[15] Wang C, Pan R, Wan X, Tan Y, Xu L, Ho CS, et al. Immediate psychological responses and associated factors during the initial stage of the 2019 Coronavirus Disease (COVID-19) epidemic among the general population in China. International Journal of Environmental Research and Public Health. 2020; 17(5):1729. [DOI:10.3390/ijerph17051729] [PMID] [PMCID]

[16] Gerard JM, Landry Meyer L, Roe JG. Grandparents raising grandchildren the role of social support in coping with caregiving challenges. International Journal of Aging \& Human Development. 2006; 62(4):359-83. [DOI:10.2190/3796-DMB2-546Q-Y4AQ] [PMID]

[17] Amarloo P, Shareh H. [Social support, responsibility, and organizational procrastination: A mediator role for basic psychological needs satisfaction (Persian)]. Iranian Journal of Psychiatry and Clinical Psychology. 2018; 24(2):176-89 [DOI:10.32598/ ijpcp.24.2.176]

[18] 18. Chadwick KA, Collins PA. Examining the relationship between social support availability, urban center size, and selfperceived mental health of recent immigrants to Canada: A mixed-methods analysis. Social Science \& Medicine (1982). 2015; 128:220-30. [DOI:10.1016/j.socscimed.2015.01.036] [PMID]

[19] Jadidi M, Safary S, Jadidi M, Jamali S. [Comparing social support and social anxiety between mothers of children with special needs and mothers of normal children (Persian)]. Knowledge \& Research in Applied Psychology. 2015; 16(2):43-52. http://jsr-p. khuisf.ac.ir/article_533965_en.html

[20] Henry A, Tourbah A, Camus G, Deschamps R, Mailhan L, Castex $\mathrm{C}$, et al. Anxiety and depression in patients with multiple sclerosis: The mediating effects of perceived social support. Multiple Sclerosis and Related Disorders. 2019; 27:46-51. [DOI:10.1016/j. msard.2018.09.039] [PMID]

[21] Kim M-Y, Johnson JL, Sawatzky R. Relationship between types of social support, coping strategies, and psychological distress in individuals living with congenital heart disease. The Journal of Cardiovascular Nursing. 2019; 34(1):76-84. [DOI:10.1097/ JCN.0000000000000531] [PMID]

[22] Bodaghi E, Alipour F, Bodaghi M, Nori R, Peiman N, Saeidpour S. [The role of spirituality and social support in pregnant women's anxiety, depression and stress symptoms (Persian)]. Journal of Community Health. 2016; 10(2):72-82. http:/ / chj.rums. ac.ir/article_45789_en.html

[23] Gunuşen NP, Wilson M, Aksoy B. Secondary traumatic stress and burnout among Muslim nurses Caring for Chronically Ill Children in a Turkish Hospital. Journal of Transcultural Nursing: Official Journal of the Transcultural Nursing Society. 2018; 29(2):146-54. [DOI:10.1177/1043659616689290] [PMID]

[24] Feng D, Su S, Wang L, Liu F. The protective role of self-esteem, perceived social support and job satisfaction against psychological distress among Chinese nurses. Journal of Nursing Management. 2018; 26(4):366-72. [DOI:10.1111/jonm.12523] [PMID]

[25] Lindblad C, Langius-Eklof A, Petersson L-M, Sackey H, Bottai M, Sandelin K. Sense of coherence is a predictor of survival: A prospective study in women treated for breast cancer. PsychoOncology. 2018; 27(6):1615-21. [DOI:10.1002/ pon.4702] [PMID] 
[26] Eriksson M, Lindstrom B. Antonovsky's sense of coherence scale and its relation with quality of life: A systematic review. Journal of Epidemiology and Community Health. 2007; 61(11):938-44 [DOI:10.1136/jech.2006.056028] [PMID] [PMCID]

[27] Urakawa K, Yokoyama K. Sense of Coherence (Soc) may reduce the effects of occupational stress on mental health status among Japanese factory workers. Industrial Health. 2009; 47(5):503-8. [DOI:10.2486/indhealth.47.503] [PMID]

[28] Kikuchi Y, Nakaya M, Ikeda M, Okuzumi S, Takeda M, Nishi M. Relationship between depressive state, job stress, and sense of coherence among female nurses. Indian Journal of Occupational and Environmental Medicine. 2014; 18(1):32-5. [DOI:10.4103/0019-5278.134959] [PMID] [PMCID]

[29] Endler PC, Haug TM, Spranger H. Sense of coherence and physical health. A copenhagen interpretation of Antonovsky's SOC concept. TheScientificWorldJournal. 2008; 8:451-3. [DOI:10.1100/ tsw.2008.59] [PMID] [PMCID]

[30] Kouvonen AM, Väänänen A, Vahtera J, Heponiemi T, Koskinen A, Cox SI, et al. Sense of coherence and psychiatric morbidity: A 19-year register-based prospective study. Journal of Epidemiology and Community Health. 2010; 64(3):255-61. [DOI:10.1136/ jech.2008.083352] [PMID]

[31] Arghabaei M, Soleimanian AA, Mohammadipour M. The role of family emotional atmosphere, sense of coherence, and affects in the prediction of tendency toward substance use among university Students. Iranian Journal of Psychiatry and Clinical Psychology. 2018; 24(3):310-23 [DOI:10.32598/ijpcp.24.3.310]

[32] Al-Said H, Braun-Lewensohn O, Sagy S. Sense of coherence, hope, and home demolition are differentially associated with anger and anxiety among Bedouin Arab adolescents in recognized and unrecognized villages. Anxiety Stress Coping. 2018; 31(4):475-85. [DOI:10.1080/10615806.2018.1454070] [PMID]

[33] del-Pino-Casado R, Espinosa-Medina A, López-Martínez C, Orgeta V. Sense of coherence, burden and mental health in caregiving: A systematic review and meta-analysis. Journal of Affective Disorders. 2019; 242:14-21. [DOI:10.1016/j.jad.2018.08.002] [PMID]

[34] Eyni S, Ebadi M, Torabi N. [Developing a model of corona anxiety in students based on optimism and resilience: The mediating role of the perceived social support (Persian)]. Counseling Culture and Psychotherapy. 2020; 11(43):1-32. [DOI:10.22054/qccpc.2020.51656.2376]

[35] Hosseinpour MR, Samiei L, Nematolahei M. [The relationship between sense of coherence and anxiety in coronary artery disease in Tabriz (Persian)]. Nursing Development in Health. 2016; 7(2):59-67. http://ndhj.lums.ac.ir/article-1-143-fa.html

[36] Puradollah M, Ghasempour M. Necessity of attention to mental health of the front line nurses against COVID-19: A forgotten requirement. International Journal of Community Based Nursing \& Midwifery. 2020; 8(3):280-1. [DOI: 10.30476/IJCBNM.2020.85889.1301]

[37] Alipour A, Ghadami A, Alipour Z, Abdollahzadeh H. [Preliminary validation of the Corona Disease Anxiety Scale (CDAS) in the Iranian sample (Persian)]. Health Psychology. 2020; 8(32):163-75. http://hpj.journals.pnu.ac.ir/article_6571. html

[38] Zimet GD, Dahlem NW, Zimet SG, Farley GK. The multidimensional scale of perceived social support. Journal of Personality Assessment. 1988; 52(1):30-41. [DOI:10.1207/s15327752jpa5201_2]
[39] Salimi A, Jokar B, Nikpour R. [Internet communication in life: Exploring the role of perception of socialsupport and loneliness in internet use (Persian)]. Quarterly Journal of Psychological Studies. 2009; 5(3):81-102. https://www.sid.ir/fa/journal/ViewPaper.aspx?id=100717

[40] Antonovsky A. The structure and properties of the sense of coherence scale. Social Science \& Medicine. 1993; 36(6):725-33. [DOI:10.1016/0277-9536(93)90033-Z]

[41] Rohani C, Khanjari S, Abedi HA, Oskouie F, Langius-Eklö A. Health Index, Sense Of Coherence Scale, Brief Religious Coping Scale and Spiritual Perspective Scale: Psychometric properties. Journal of Advanced Nursing. 2010; 66(12):2796-806. [DOI:10.1111/j.1365-2648.2010.05409.x] [PMID]

[42] Lustig DC, Rosenthal DA, Strauser DR, Haynes K. The relationship between sense of coherence and adjustment in persons with disabilities. Rehabilitation Counseling Bulletin. 2000; 43(3):134-41. [DOI:10.1177/003435520004300302]

[43] Mahammadzadeh A, Poursharifi H, Alipour A. Validation of Sense of Coherence (SOC) 13 - item scale in Iranian sample. Procedia-Social and Behavioral Sciences. 2010; 5:1451-5. [DOI:10.1016/j. sbspro.2010.07.306] 